\title{
PARISIAN RUIN OF THE BROWNIAN MOTION RISK MODEL WITH CONSTANT FORCE OF INTEREST
}

LONG BAI AND LI LUO

Abstract: Let $B(t), t \in \mathbb{R}$ be a standard Brownian motion. Define a risk process

$$
R_{u}^{\delta}(t)=e^{\delta t}\left(u+c \int_{0}^{t} e^{-\delta s} d s-\sigma \int_{0}^{t} e^{-\delta s} d B(s)\right), t \geq 0
$$

where $u \geq 0$ is the initial reserve, $\delta \geq 0$ is the force of interest, $c>0$ is the rate of premium and $\sigma>0$ is a volatility factor. In this contribution we obtain an approximation of the Parisian ruin probability

$$
\mathcal{K}_{S}^{\delta}\left(u, T_{u}\right):=\mathbb{P}\left\{\inf _{t \in[0, S]} \sup _{s \in\left[t, t+T_{u}\right]} R_{u}^{\delta}(s)<0\right\}, S \geq 0,
$$

as $u \rightarrow \infty$ where $T_{u}$ is a bounded function. Further, we show that the Parisian ruin time of this risk process can be approximated by an exponential random variable. Our results are new even for the classical ruin probability and ruin time which correspond to $T_{u} \equiv 0$ in the Parisian setting.

Key Words: Parisian ruin; ruin probability; ruin time; Brownian motion

AMS Classification: Primary 60G15; secondary 60G70

\section{INTRODUCTION}

In a theoretical insurance model the surplus process $R_{u}(t)$ can be defined by

$$
R_{u}(t)=u+c t-X(t), \quad t \geq 0
$$

see [10], where $u \geq 0$ is the initial reserve, $c>0$ is the rate of premium and $X(t), t \geq 0$ denotes the aggregate claims process. More specifically, we assume that the aggregate claims process is a Brownian motion, i.e., $X(t)=\sigma B(t), \sigma>$ 0 . Due to the nature of the financial market, we shall consider a more general surplus process including interest rate, see [18], called a risk reserve process with constant force of interest, i.e., $R_{u}^{\delta}(t), t \geq 0$, in (0.1). See [18, 3, 14] for more studies on risk models with force of interest.

During the time horizon $[0, S], S \in(0, \infty]$, the classical ruin probability is defined as below

$$
\psi_{S}^{\delta}(u):=\mathbb{P}\left\{\inf _{t \in[0, S]} R_{u}^{\delta}(t)<0\right\}
$$

see $[10,15,16,8]$. In $[9,11]$ the exact formula of $\psi_{\infty}^{\delta}(u)$ for $\delta>0$ is shown to be

$$
\psi_{\infty}^{\delta}(u)=\frac{\Psi\left(\sqrt{\frac{2 \delta}{\sigma^{2}}} u+\sqrt{\frac{2 c^{2}}{\sigma^{2} \delta}}\right)}{\Psi\left(\sqrt{\frac{2 c^{2}}{\sigma^{2} \delta}}\right)}, u>0,
$$

where $\Psi(x)=1-\Phi(x)$ with $\Phi(\cdot)$ the distribution function of an $\mathcal{N}(0,1)$ random variable.

For $\delta=0$, the exact value of $\psi_{\infty}^{0}(u)$ is well-known (cf. [7]) with

$$
\psi_{\infty}^{0}(u)=e^{-\frac{2 c u}{\sigma^{2}}}, \quad u>0 .
$$

1

(C) 2016. This manuscript version is made available under the Elsevier user license

http://www.elsevier.com/open-access/userlicense/1.0/ 
In the literature, there are no results for the classical ruin probability in the case of finite time horizon, i.e., $S \in(0, \infty)$. For $S \in(0, \infty)$, with motivation from the recent contributions $[4,5]$ we shall investigate in this paper the Parisian ruin probability over the time period $[0, S]$ defined as

$$
\mathcal{K}_{S}^{\delta}\left(u, T_{u}\right):=\mathbb{P}\left\{\inf _{t \in[0, S]} \sup _{s \in\left[t, t+T_{u}\right]} R_{u}^{\delta}(s)<0\right\},
$$

where $T_{u} \geq 0$ models the pre-specified time. Our assumption on $T_{u}$ is that

$$
\lim _{u \rightarrow \infty} T_{u} u^{2}=T \in[0, \infty)
$$

and thus $\psi_{S}^{\delta}(u)$ is a special case of $\mathcal{K}_{S}^{\delta}\left(u, T_{u}\right)$ with $T_{u} \equiv 0$.

Another quantity of interest is the conditional distribution of the ruin time for the surplus process $R_{u}^{\delta}(t)$. The classical ruin time, e.g., $[3,12,16]$, is defined as

$$
\tau(u)=\inf \left\{t>0: R_{u}^{\delta}(t)<0\right\} .
$$

Here as in [4] we define the Parisian ruin time of the risk process $R_{u}^{\delta}(t)$ by

$$
\eta(u)=\inf \left\{t \geq T_{u}: t-\kappa_{t, u} \geq T_{u}, R_{u}^{\delta}(t)<0\right\}, \quad \text { with } \kappa_{t, u}=\sup \left\{s \in[0, t]: R_{u}^{\delta}(s) \geq 0\right\},
$$

and $\tau(u)$ is a special case of $\eta(u)$ with $T_{u} \equiv 0$.

Brief organization of the rest of the paper: In Section 2 we first present our main results on the asymptotics of $\mathcal{K}_{S}^{\delta}\left(u, T_{u}\right)$ as $u \rightarrow \infty$ and then we display the approximation of the Parisian ruin time. All the proofs are relegated to Section 3 .

\section{MAin Results}

Before giving the main results, we shall introduce a generalized Piterbarg constant as

$$
\widetilde{\mathcal{P}}(T)=\lim _{\lambda \rightarrow \infty} \widetilde{\mathcal{P}}(\lambda, T), \quad T \geq 0,
$$

where for $\lambda, T \geq 0$

$$
\widetilde{\mathcal{P}}(\lambda, T)=\mathbb{E}\left\{\sup _{t \in[0, \lambda]} \inf _{s \in[0, T]} e^{\sqrt{2} B(t-s)-|t-s|-(t-s)}\right\} .
$$

Note further that the classical Piterbarg constant $\mathcal{P}_{1}^{1}[0, \infty)$ equals $\widetilde{\mathcal{P}}(0)$ and $\mathcal{P}_{1}^{1}[0, \infty)=2$, see $[6,1,13]$.

Through this paper $\sim$ means asymptotic equivalence when the argument tends to 0 or $\infty$. Recall that $\Psi(\cdot)$ denotes the tail distribution function of an $\mathcal{N}(0,1)$ random variable and $\Psi(u) \sim \frac{1}{\sqrt{2 \pi} u} e^{-\frac{u^{2}}{2}}, u \rightarrow \infty$.

Theorem 2.1. For $\delta>0, S>0$ and $\lim _{u \rightarrow \infty} T_{u} u^{2}=T \in[0, \infty)$, we have

$$
\mathcal{K}_{S}^{\delta}\left(u, T_{u}\right) \sim \widetilde{\mathcal{P}}(a T) \Psi\left(\frac{\sqrt{2 \delta}\left(u+\frac{c}{\delta}\left(1-e^{-\delta S}\right)\right)}{\sigma \sqrt{1-e^{-2 \delta S}}}\right), u \rightarrow \infty,
$$

where $a:=\frac{2 \delta^{2} e^{-2 \delta S}}{\sigma^{2}\left(1-e^{-2 \delta S}\right)^{2}}$.

Remarks 2.2. a) When $T_{u} \equiv 0, \mathcal{K}_{S}^{\delta}\left(u, T_{u}\right)$ reduces to the classical ruin probability $\psi_{S}^{\delta}(u)$, and by Theorem 2.1 with $T=0$

$$
\mathcal{K}_{S}^{\delta}(u, 0)=\psi_{S}^{\delta}(u) \sim 2 \Psi\left(\frac{\sqrt{2 \delta}\left(u+\frac{c}{\delta}\left(1-e^{-\delta S}\right)\right)}{\sigma \sqrt{1-e^{-2 \delta S}}}\right), u \rightarrow \infty
$$


b) If $\delta=0$

$$
\begin{aligned}
\mathcal{K}_{S}^{0}\left(u, T_{u}\right) & =\mathbb{P}\left\{\inf _{t \in[0, S]} \sup _{s \in\left[t, t+T_{u}\right]}(u+c s-\sigma B(s))<0\right\} \\
& \sim \widetilde{\mathcal{P}}(b T) \Psi\left(\frac{u+c S}{\sigma \sqrt{S}}\right), u \rightarrow \infty,
\end{aligned}
$$

where $b:=\frac{1}{2 \sigma^{2} S^{2}}$ and we used the result of Corollary 3.4 (ii) in [5].

Further, if $\delta=0$ and $T_{u} \equiv 0$, by (2.3) with $T=0$, we get the asymptotic result of the classical ruin probability

$$
\psi_{S}^{0}(u) \sim 2 \Psi\left(\frac{u+c S}{\sigma \sqrt{S}}\right), u \rightarrow \infty .
$$

In fact, [7] gave the exact result of $\psi_{S}^{0}(u), u>0$, i.e.,

$$
\begin{aligned}
\psi_{S}^{0}(u) & =\Psi\left(\frac{u+c S}{\sigma \sqrt{S}}\right)+e^{-\frac{2 c u}{\sigma^{2}}} \Phi\left(\frac{c S-u}{\sigma \sqrt{S}}\right) \\
& \sim 2 \Psi\left(\frac{u+c S}{\sigma \sqrt{S}}\right), u \rightarrow \infty
\end{aligned}
$$

which follows from

$$
\lim _{u \rightarrow \infty} \frac{e^{-\frac{2 c u}{\sigma^{2}}} \Phi\left(\frac{c S-u}{\sigma \sqrt{S}}\right)}{\Psi\left(\frac{u+c S}{\sigma \sqrt{S}}\right)}=\lim _{u \rightarrow \infty} \frac{-\frac{2 c}{\sigma^{2}} e^{-\frac{2 c u}{\sigma^{2}}} \Phi\left(\frac{c S-u}{\sigma \sqrt{S}}\right)-\frac{1}{\sigma \sqrt{2 \pi S}} e^{-\left(\frac{u+c S}{\sigma \sqrt{S}}\right)^{2} / 2}}{-\frac{1}{\sigma \sqrt{2 \pi S}} e^{-\left(\frac{u+c S}{\sigma \sqrt{S}}\right)^{2} / 2}}=1 .
$$

Our next result discusses the approximation of the conditional ruin time.

Theorem 2.3. Let $\eta(u)$ satisfy (1.4), under the assumptions of Theorem 2.1, we have for any $x>0$ and $\delta \geq 0$,

$$
\mathbb{P}\left\{u^{2}\left(S+T_{u}-\eta(u)\right)>x \mid \eta(u) \leq S+T_{u}\right\} \sim\left\{\begin{array}{ll}
\exp (-a x), & \text { if } \delta>0, \\
\exp (-b x), & \text { if } \delta=0,
\end{array} u \rightarrow \infty,\right.
$$

where $a:=\frac{2 \delta^{2} e^{-2 \delta S}}{\sigma^{2}\left(1-e^{-2 \delta S}\right)^{2}}$ and $b:=\frac{1}{2 \sigma^{2} S^{2}}$.

Remark 2.4. If $T_{u} \equiv 0$, then $\eta(u)=\tau(u)$ and by Theorem 2.3, we obtain as $u \rightarrow \infty$

$$
\mathbb{P}\left\{u^{2}(S-\tau(u))>x \mid \tau(u) \leq S\right\} \sim \begin{cases}\exp (-a x), & \text { if } \delta>0, \\ \exp (-b x), & \text { if } \delta=0 .\end{cases}
$$

\section{Proofs}

Hereafter we assume that $\mathbb{C}_{i}, i \in \mathbb{N}$ are some positive constants.

Proof of Theorem 2.1 For $S>0$ and $u$ large enough

$$
\begin{aligned}
\mathcal{K}_{S}^{\delta}\left(u, T_{u}\right) & =\mathbb{P}\left\{\sup _{t \in[0, S]} \inf _{s \in\left[t, t+T_{u}\right]}\left(\sigma \int_{0}^{s} e^{-\delta z} d B(z)-c \int_{0}^{s} e^{-\delta z} d z\right)>u\right\} \\
& =\mathbb{P}\left\{\sup _{t \in[0, S]} \inf _{s \in\left[t, t+T_{u}\right]} \bar{X}(s) \frac{f_{u}(S)}{f_{u}(s)}>f_{u}(S)\right\} \\
& =\mathbb{P}\left\{\sup _{t \in[0, S]} \inf _{s \in\left[t, t+T_{u}\right]} X_{u}(s)>f_{u}(S)\right\},
\end{aligned}
$$

with

$$
X(s)=\sigma \int_{0}^{s} e^{-\delta z} d B(z), \bar{X}(s)=\frac{X(s)}{\sigma_{X}(s)}, f_{u}(s)=\frac{u+\frac{c}{\delta}\left(1-e^{-\delta s}\right)}{\sigma_{X}(s)} \text { and } X_{u}(s)=\bar{X}(s) \frac{f_{u}(S)}{f_{u}(s)}
$$


where $\sigma_{X}^{2}(s)$ is the variance of $X(s)$ with $\sigma_{X}^{2}(s)=\frac{\sigma^{2}}{2 \delta}\left(1-e^{-2 \delta s}\right)$.

Set $\rho(u)=\left(\frac{\ln u}{u}\right)^{2}$ and for any $\lambda>0$, Bonferroni inequality yields

$$
\Pi_{0}(u):=\mathbb{P}\left\{\sup _{t \in\left[S-\lambda u^{-2}, S\right]} \inf _{s \in\left[t, t+T_{u}\right]} X_{u}(s)>f_{u}(S)\right\} \leq \mathcal{K}_{S}^{\delta}\left(u, T_{u}\right) \leq \Pi_{0}(u)+\Pi_{1}(u)+\Pi_{2}(u),
$$

where

$$
\Pi_{1}(u)=\mathbb{P}\left\{\sup _{t \in[0, S-\rho(u)]} \inf _{s \in\left[t, t+T_{u}\right]} X_{u}(s)>f_{u}(S)\right\}, \quad \Pi_{2}(u)=\mathbb{P}\left\{\sup _{t \in\left[S-\rho(u), S-\lambda u^{-2}\right]} \inf _{s \in\left[t, t+T_{u}\right]} X_{u}(s)>f_{u}(S)\right\} .
$$

First we give some upper bounds of $\Pi_{1}(u)$ and $\Pi_{2}(u)$ which finally show that

$$
\Pi_{1}(u)+\Pi_{2}(u)=o\left(\Pi_{0}(u)\right), \quad u \rightarrow \infty .
$$

For all $u$ large

$$
\begin{aligned}
\mathbb{E}\left\{\left(X_{u}\left(t_{1}\right)-X_{u}\left(t_{2}\right)\right)^{2}\right\} & =\mathbb{E}\left\{\left(X\left(t_{1}\right) \frac{f_{u}(S)}{u+\frac{c}{\delta}\left(1-e^{-\delta t_{1}}\right)}-X\left(t_{2}\right) \frac{f_{u}(S)}{u+\frac{c}{\delta}\left(1-e^{-\delta t_{2}}\right)}\right)^{2}\right\} \\
& \leq \mathbb{C}_{1} \mathbb{E}\left\{\left(\int_{t_{1}}^{t_{2}} e^{-\delta z} d B(z)\right)^{2}\right\}+\mathbb{C}_{2}\left(\frac{u+\frac{c}{\delta}\left(1-e^{-\delta S}\right)}{u+\frac{c}{\delta}\left(1-e^{-\delta t_{1}}\right)}-\frac{u+\frac{c}{\delta}\left(1-e^{-\delta S}\right)}{u+\frac{c}{\delta}\left(1-e^{-\delta t_{2}}\right)}\right)^{2} \\
& \leq \mathbb{C}_{3}\left|t_{1}-t_{2}\right|, \quad t_{1}<t_{2}, t_{1}, t_{2} \in(0, S] .
\end{aligned}
$$

Moreover,

$$
\sup _{t \in[0, S-\rho(u)]} \operatorname{Var}\left(X_{u}(t)\right)=\sup _{t \in[0, S-\rho(u)]}\left(\frac{f_{u}(S)}{f_{u}(t)}\right)^{2}=\frac{f_{u}^{2}(S)}{f_{u}^{2}(S-\rho(u))},
$$

where we use the fact that $f_{u}(t)$ is a decreasing function for $t \in[0, S]$ when $u$ large enough. Therefore, by Theorem 8.1 in $[17]$, we obtain

$$
\Pi_{1}(u) \leq \mathbb{P}\left\{\sup _{t \in[0, S-\rho(u)]} X_{u}(t)>f_{u}(S)\right\} \leq \mathbb{C}_{4} u^{2} \Psi\left(f_{u}(S-\rho(u))\right),
$$

and direct calculation yields that

$$
\begin{aligned}
u^{2} \Psi\left(f_{u}(S-\rho(u))\right) & \leq \frac{u^{2}}{\sqrt{2 \pi} f_{u}(S)} e^{-\frac{f_{u}^{2}(S)}{2}\left(\frac{f_{u}^{2}(S-\rho(u))}{f_{u}^{2}(S)}-1\right)} e^{-\frac{f_{u}^{2}(S)}{2}} \\
& \sim u^{2} e^{-a(\ln u)^{2}} \Psi\left(f_{u}(S)\right)=o\left(\Psi\left(f_{u}(S)\right)\right), \quad u \rightarrow \infty,
\end{aligned}
$$

where $a=\frac{2 \delta^{2} e^{-2 \delta S}}{\sigma^{2}\left(1-e^{-2 \delta S}\right)^{2}}$ and we use the fact that

$$
1-\frac{f_{u}(S)}{f_{u}(S-t)} \sim \frac{\delta e^{-2 \delta S}}{1-e^{-2 \delta S}} t, \quad t \rightarrow 0 .
$$

Set

$$
\triangle_{k}=\left[k \lambda u^{-2},(k+1) \lambda u^{-2}\right], k \in \mathbb{N}, \text { and } N(u)=\left\lfloor\lambda^{-1} \rho(u) u^{2}\right\rfloor,
$$

where $\lfloor\cdot\rfloor$ stands for the ceiling function, then

$$
\begin{aligned}
\Pi_{2}(u) & \leq \mathbb{P}\left\{\sup _{t \in\left[S-\rho(u), S-\lambda u^{-2}\right]} X_{u}(t)>f_{u}(S)\right\} \\
& =\mathbb{P}\left\{\sup _{t \in\left[\lambda u^{-2}, \rho(u)\right]} X_{u}(S-t)>f_{u}(S)\right\} \\
& \leq \sum_{k=1}^{N(u)} \mathbb{P}\left\{\sup _{t \in \Delta_{k}} X_{u}(S-t)>f_{u}(S)\right\}
\end{aligned}
$$




$$
\begin{aligned}
& \leq \sum_{k=1}^{N(u)} \mathbb{P}\left\{\sup _{t \in \Delta_{0}} \bar{X}(S-t)>f_{u}\left(S-k \lambda u^{-2}\right)\right\} \\
& \leq \sum_{k=1}^{N(u)} \mathbb{P}\left\{\sup _{t \in[0, \lambda]} \bar{X}\left(S-u^{-2} t\right)>f_{u}\left(S-k \lambda u^{-2}\right)\right\} .
\end{aligned}
$$

Clearly,

$$
\inf _{1 \leq k \leq N(u)} f_{u}\left(S-k \lambda u^{-2}\right) \rightarrow \infty, u \rightarrow \infty
$$

and for $t_{1}<t_{2}, t_{1}, t_{2} \in[0, S]$,

$$
r_{X}\left(t_{1}, t_{2}\right):=\mathbb{E}\left\{\bar{X}\left(t_{1}\right) \bar{X}\left(t_{2}\right)\right\}=\sqrt{\frac{1-e^{-2 \delta t_{1}}}{1-e^{-2 \delta t_{2}}}} .
$$

Further,

$$
\begin{aligned}
& \lim _{u \rightarrow \infty} \sup _{1 \leq k \leq N(u)} \sup _{\substack{t_{1} \neq t_{2}, t_{1}, t_{2} \in[0, \lambda]}}\left|f_{u}^{2}\left(S-k \lambda u^{-2}\right) \frac{\operatorname{Var}\left(\bar{X}\left(S-u^{-2} t_{1}\right)-\bar{X}\left(S-u^{-2} t_{2}\right)\right)}{2 a\left|t_{1}-t_{2}\right|}-1\right| \\
& \quad=\lim _{u \rightarrow \infty} \sup _{1 \leq k \leq N(u)} \sup _{\substack{1 \neq t_{2}, t_{1}, t_{2} \in[0, \lambda]}}\left|f_{u}^{2}\left(S-k \lambda u^{-2}\right) \frac{2-2 r_{X}\left(S-u^{-2} t_{1}, S-u^{-2} t_{2}\right)}{2 a\left|t_{1}-t_{2}\right|}-1\right| \\
& \quad=0
\end{aligned}
$$

and

$$
\begin{aligned}
& \sup _{1 \leq k \leq N(u)} \sup _{\left|t_{1}-t_{2}\right|<\varepsilon} f_{u}^{2}\left(S-k \lambda u^{-2}\right) \mathbb{E}\left\{\left(\bar{X}\left(S-u^{-2} t_{1}\right)-\bar{X}\left(S-u^{-2} t_{2}\right)\right) \bar{X}(S)\right\} \\
& t_{1}, t_{2} \in[0, \lambda] \\
& \leq \mathbb{C}_{5} u^{2} \sup _{\substack{\left|t_{1}-t_{2}\right|<\varepsilon \\
t_{1}, t_{2} \in[0, \lambda]}}\left|r_{X}\left(S-u^{-2} t_{1}, S\right)-r_{X}\left(S-u^{-2} t_{2}, S\right)\right| \\
& \leq \mathbb{C}_{6} u^{2} \sup _{\substack{\left|t_{1}-t_{2}\right|<\varepsilon \\
t_{1}, t_{2} \in[0, \lambda]}}\left|\sqrt{1-e^{-2 \delta\left(S-u^{-2} t_{1}\right)}}-\sqrt{1-e^{-2 \delta\left(S-u^{-2} t_{2}\right)}}\right| \\
& \leq \mathbb{C}_{7} \sup _{\substack{\left|t_{1}-t_{2}\right|<\varepsilon \\
t_{1}, t_{2} \in[0, \lambda]}}\left|t_{1}-t_{2}\right| \rightarrow 0, \quad u \rightarrow \infty, \varepsilon \rightarrow 0 .
\end{aligned}
$$

According to (3.6), (3.7), (3.8) and Lemma 5.3 of [2], (3.5) is followed by

$$
\Pi_{2}(u) \leq \mathbb{C}_{8} \lambda \sum_{k=1}^{N(u)} \Psi\left(f_{u}\left(S-k \lambda u^{-2}\right)\right) \leq \mathbb{C}_{9} \Psi\left(f_{u}(S)\right) \lambda \sum_{k=1}^{\infty} e^{-\mathbb{C}_{10} k \lambda}=o\left(\Psi\left(f_{u}(S)\right)\right), u \rightarrow \infty, \lambda \rightarrow \infty,
$$

where the last inequality follows from (3.4).

Next we give the asymptotic behavior of $\Pi_{0}(u)$ as $u \rightarrow \infty$ based on an appropriate application of the Appendix in [5].

For any $\varepsilon_{1}>0$ and $u$ large enough

$$
\begin{aligned}
\Pi_{0}(u) & =\mathbb{P}\left\{\sup _{t \in\left[S-\lambda u^{-2}, S\right]} \inf _{s \in\left[t, t+T_{u}\right]} X_{u}(s)>f_{u}(S)\right\} \\
& \leq \mathbb{P}\left\{\sup _{t \in\left[S-\lambda u^{-2}, S\right]} \inf _{s \in\left[t, t+\left(1-\varepsilon_{1}\right) T u^{-2}\right]} X_{u}(s)>f_{u}(S)\right\} \\
& =\mathbb{P}\left\{\sup _{t \in[0, \lambda]} \inf _{s \in\left[0,\left(1-\varepsilon_{1}\right) T\right]} X_{u}\left(S+u^{-2} s-u^{-2} t\right)>f_{u}(S)\right\}
\end{aligned}
$$




$$
\begin{aligned}
& =\mathbb{P}\left\{\sup _{t \in[0, \lambda]} \inf _{s \in\left[0,\left(1-\varepsilon_{1}\right) T\right]} Y_{u}(t, s)>f_{u}(S)\right\} \\
& =: \Pi_{0}^{+}(u)
\end{aligned}
$$

and

$$
\Pi_{0}(u) \geq \mathbb{P}\left\{\sup _{t \in[0, \lambda]} \inf _{s \in\left[0,\left(1+\varepsilon_{1}\right) T\right]} Y_{u}(t, s)>f_{u}(S)\right\}=: \Pi_{0}^{-}(u),
$$

where $Y_{u}(t, s):=X_{u}\left(S+u^{-2} s-u^{-2} t\right)$, for $(t, s) \in[0, \lambda] \times\left[0,\left(1+\varepsilon_{1}\right) T\right]$.

Since

$$
\sigma_{Y_{u}}(t, s):=\sqrt{\operatorname{Var}\left(Y_{u}(t, s)\right)}=\sqrt{\operatorname{Var}\left(X_{u}\left(S+u^{-2} s-u^{-2} t\right)\right)}=\frac{f_{u}(S)}{f_{u}\left(S+u^{-2} s-u^{-2} t\right)}
$$

and (3.4), there exists $d(t, s)=\frac{\delta e^{-2 \delta S}}{1-e^{-2 \delta S}}(t-s)$ such that

$$
\lim _{u \rightarrow \infty} \sup _{(t, s) \in[0, \lambda] \times\left[0,\left(1+\varepsilon_{1}\right) T\right]}\left|u^{2}\left(1-\sigma_{Y_{u}}(t, s)\right)-d(t, s)\right|=0 .
$$

Moreover, for $\left(t_{1}, s_{1}\right),\left(t_{2}, s_{2}\right) \in[0, \lambda] \times\left[0,\left(1+\varepsilon_{1}\right) T\right]$ and $s_{1}-t_{1}>s_{2}-t_{2}$,

$$
\begin{aligned}
\operatorname{Var} & \left(Y_{u}\left(t_{1}, s_{1}\right)-Y_{u}\left(t_{2}, s_{2}\right)\right) \\
& =f_{u}^{2}(S) \mathbb{E}\left\{\frac{X\left(S+u^{-2} s_{1}-u^{-2} t_{1}\right)}{u+\frac{c}{\delta}\left(1-e^{-\delta\left(S+u^{-2} s_{1}-u^{-2} t_{1}\right)}\right)}-\frac{X\left(S+u^{-2} s_{2}-u^{-2} t_{2}\right)}{u+\frac{c}{\delta}\left(1-e^{-\delta\left(S+u^{-2} s_{2}-u^{-2} t_{2}\right)}\right)}\right\}^{2} \\
& =f_{u}^{2}(S)\left(J_{1}(u)+J_{2}(u)+J_{3}(u)\right),
\end{aligned}
$$

where

$$
\begin{aligned}
J_{1}(u)= & \mathbb{E}\left\{\frac{X\left(S+u^{-2} s_{1}-u^{-2} t_{1}\right)-X\left(S+u^{-2} s_{2}-u^{-2} t_{2}\right)}{u+\frac{c}{\delta}\left(1-e^{-\delta\left(S+u^{-2} s_{1}-u^{-2} t_{1}\right)}\right)}\right\}^{2}, \\
J_{2}(u)= & 2 \frac{\frac{c}{\delta}\left(e^{-\delta\left(S+u^{-2} s_{1}-u^{-2} t_{1}\right)}-e^{-\delta\left(S+u^{-2} s_{2}-u^{-2} t_{2}\right)}\right)}{\left(u+\frac{c}{\delta}\left(1-e^{-\delta\left(S+u^{-2} s_{1}-u^{-2} t_{1}\right)}\right)\right)\left(u+\frac{c}{\delta}\left(1-e^{-\delta\left(S+u^{-2} s_{2}-u^{-2} t_{2}\right)}\right)\right)} \\
& \times \mathbb{E}\left\{\left(\frac{X\left(S+u^{-2} s_{1}-u^{-2} t_{1}\right)-X\left(S+u^{-2} s_{2}-u^{-2} t_{2}\right)}{u+\frac{c}{\delta}\left(1-e^{-\delta\left(S+u^{-2} s_{1}-u^{-2} t_{1}\right)}\right)}\right) X\left(S+u^{-2} s_{2}-u^{-2} t_{2}\right)\right\}=0, \\
J_{3}(u)= & \left(\frac{\frac{c}{\delta}\left(e^{-\delta\left(S+u^{-2} s_{1}-u^{-2} t_{1}\right)}-e^{-\delta\left(S+u^{-2} s_{2}-u^{-2} t_{2}\right)}\right)}{\left(u+\frac{c}{\delta}\left(1-e^{-\delta\left(S+u^{-2} s_{1}-u^{-2} t_{1}\right)}\right)\right)\left(u+\frac{c}{\delta}\left(1-e^{-\delta\left(S+u^{-2} s_{2}-u^{-2} t_{2}\right)}\right)\right)}\right)^{2} \mathbb{E}\left\{X\left(S+u^{-2} s_{2}-u^{-2} t_{2}\right)\right\}^{2} .
\end{aligned}
$$

Since

$$
\begin{aligned}
\lim _{u \rightarrow \infty} u^{2} f_{u}^{2}(S) J_{1}(u) & =\lim _{u \rightarrow \infty} f_{u}^{2}(S) \mathbb{E}\left\{X\left(S+u^{-2} s_{1}-u^{-2} t_{1}\right)-X\left(S+u^{-2} s_{2}-u^{-2} t_{2}\right)\right\}^{2} \\
& =\lim _{u \rightarrow \infty} \frac{u^{2}}{\frac{\sigma^{2}}{2 \delta}\left(1-e^{-2 \delta S}\right)} \frac{\sigma^{2}}{2 \delta}\left(e^{-2 \delta\left(S+u^{-2} s_{2}-u^{-2} t_{2}\right)}-e^{-2 \delta\left(S+u^{-2} s_{1}-u^{-2} t_{1}\right)}\right) \\
& =\frac{2 \delta e^{-2 \delta S}}{1-e^{-2 \delta S}}\left(\left(s_{1}-s_{2}\right)-\left(t_{1}-t_{2}\right)\right) \\
& =\frac{2 \delta e^{-2 \delta S}}{1-e^{-2 \delta S}} \operatorname{Var}\left(B\left(s_{1}-t_{1}\right)-B\left(s_{2}-t_{2}\right)\right),
\end{aligned}
$$

$\lim _{u \rightarrow \infty} u^{2} f_{u}^{2}(S) J_{3}(u) \leq \lim _{u \rightarrow \infty} \mathbb{C}_{11}\left(e^{-\delta\left(S+u^{-2} s_{1}-u^{-2} t_{1}\right)}-e^{-\delta\left(S+u^{-2} s_{2}-u^{-2} t_{2}\right)}\right) \mathbb{E}\left\{X\left(S+u^{-2} s_{2}-u^{-2} t_{2}\right)\right\}^{2}=0$,

thus

$$
\lim _{u \rightarrow \infty} u^{2} \operatorname{Var}\left(Y_{u}\left(t_{1}, s_{1}\right)-Y_{u}\left(t_{2}, s_{2}\right)\right)=\frac{2 \delta e^{-2 \delta S}}{1-e^{-2 \delta S}} \operatorname{Var}\left(B\left(s_{1}-t_{1}\right)-B\left(s_{2}-t_{2}\right)\right)
$$


Further, there exist some constant $G, u_{0}>0$, such that for any $u>u_{0}$

$$
u^{2} \operatorname{Var}\left(Y_{u}\left(t_{1}, s_{1}\right)-Y_{u}\left(t_{2}, s_{2}\right)\right) \leq G\left(\left|t_{1}-t_{2}\right|+\left|s_{1}-s_{2}\right|\right)
$$

holds uniformly with respect to $\left(t_{1}, s_{1}\right),\left(t_{2}, s_{2}\right) \in[0, \lambda] \times\left[0,\left(1+\varepsilon_{1}\right) T\right]$. By $(3.10),(3.11),(3.12)$, Lemma 5.1 in [5] and $\lim _{u \rightarrow \infty} f_{u}(S) / u=1 / \sigma_{X}(S)$, we obtain

$$
\Pi_{0}^{-}(u) \sim \widetilde{\mathcal{P}}\left(a \lambda, a\left(1+\varepsilon_{1}\right) T\right) \Psi\left(f_{u}(S)\right), u \rightarrow \infty
$$

Similarly

$$
\Pi_{0}^{+}(u) \sim \widetilde{\mathcal{P}}\left(a \lambda, a\left(1-\varepsilon_{1}\right) T\right) \Psi\left(f_{u}(S)\right), u \rightarrow \infty
$$

Letting $\varepsilon_{1} \rightarrow 0$ and $\lambda \rightarrow \infty$, we have

$$
\Pi_{0}(u) \sim \widetilde{\mathcal{P}}(a T) \Psi\left(f_{u}(S)\right), u \rightarrow \infty
$$

The above combined with (3.3) and (3.9) drives (3.2), therefore by (3.1) the proof is complete.

Proof of Theorem 2.3 Case $1 \delta>0$ : According to the definition of conditional probability, for any $x, u>0$

$$
\begin{aligned}
\mathbb{P}\left\{u^{2}\left(S+T_{u}-\eta(u)\right)>x \mid \eta(u) \leq S+T_{u}\right\} \\
=\frac{\mathbb{P}\left\{\sup _{t \in\left[0, S-x u^{-2}\right]} \inf _{s \in\left[t, t+T_{u}\right]}\left(\sigma \int_{0}^{s} e^{-\delta z} d B(z)-c \int_{0}^{s} e^{-\delta z} d z\right)>u\right\}}{\mathbb{P}\left\{\sup _{t \in[0, S]} \inf _{s \in\left[t, t+T_{u}\right]}\left(\sigma \int_{0}^{s} e^{-\delta z} d B(z)-c \int_{0}^{s} e^{-\delta z} d z\right)>u\right\}} .
\end{aligned}
$$

Using the same notation of $X(s), \bar{X}(s), f_{u}(s), X_{u}(s), \sigma_{X}(s)$ as in the proof of Theorem 2.1, we have for $u$ large enough

$$
\begin{aligned}
\mathbb{P}\left\{\sup _{t \in\left[0, S-x u^{-2}\right]} \inf _{s \in\left[t, t+T_{u}\right]}\left(\sigma \int_{0}^{s} e^{-\delta z} d B(z)-c \int_{0}^{s} e^{-\delta z} d z\right)>u\right\} \\
=\mathbb{P}\left\{\sup _{t \in\left[0, S-x u^{-2}\right]} \inf _{s \in\left[t, t+T_{u}\right]} \bar{X}(s) \frac{f_{u}(S)}{f_{u}(s)}>f_{u}(S)\right\} \\
=\mathbb{P}\left\{\sup _{t \in\left[0, S-x u^{-2}\right]} \inf _{s \in\left[t, t+T_{u}\right]} X_{u}(s)>f_{u}(S)\right\},
\end{aligned}
$$

Set $\rho(u)=\left(\frac{\ln u}{u}\right)^{2}$. For any $\lambda>0$, Bonferroni inequality yields

$$
\Pi_{0}^{*}(u) \leq \mathbb{P}\left\{\sup _{t \in\left[0, S-x u^{-2}\right]} \inf _{s \in\left[t, t+T_{u}\right]} X_{u}(s)>f_{u}(S)\right\} \leq \Pi_{0}^{*}(u)+\Pi_{1}^{*}(u)+\Pi_{2}^{*}(u),
$$

where

$$
\begin{aligned}
& \Pi_{0}^{*}(u)=\mathbb{P}\left\{\sup _{t \in\left[S-x u^{-2}-\lambda u^{-2}, S-x u^{-2}\right]} \inf _{s \in\left[t, t+T_{u}\right]} X_{u}(s)>f_{u}(S)\right\}, \\
& \Pi_{1}^{*}(u)=\mathbb{P}\left\{\sup _{t \in[0, S-\rho(u)]} \inf _{s \in\left[t, t+T_{u}\right]} X_{u}(s)>f_{u}(S)\right\}, \\
& \Pi_{2}^{*}(u)=\mathbb{P}\left\{\sup _{t \in\left[S-\rho(u), S-x u^{-2}-\lambda u^{-2}\right]} \inf _{s \in\left[t, t+T_{u}\right]} X_{u}(s)>f_{u}(S)\right\} .
\end{aligned}
$$

By (3.3) and (3.9) in the proof of Theorem 2.1, we know

$$
\Pi_{1}^{*}(u)=o\left(\Psi\left(f_{u}(S)\right)\right), \quad u \rightarrow \infty,
$$


and

$$
\Pi_{2}^{*}(u) \leq \mathbb{P}\left\{\sup _{t \in\left[S-\rho(u), S-\lambda u^{-2}\right]} \inf _{s \in\left[t, t+T_{u}\right]} X_{u}(s)>f_{u}(S)\right\}=o\left(\Psi\left(f_{u}(S)\right)\right), u \rightarrow \infty, \lambda \rightarrow \infty .
$$

Next we give the asymptotic behavior of $\Pi_{0}^{*}(u)$ as $u \rightarrow \infty$. For any $\varepsilon_{1}>0$ and $u$ large enough

$$
\begin{aligned}
& \Pi_{0}^{*}(u)=\mathbb{P}\left\{\sup _{t \in\left[S-x u^{-2}-\lambda u^{-2}, S-x u^{-2}\right]} \inf _{s \in\left[t, t+T_{u}\right]} \bar{X}(s) \frac{f_{u}(S)}{f_{u}(s)}>f_{u}(S)\right\} \\
& =\mathbb{P}\left\{\sup _{t \in\left[S-x u^{-2}-\lambda u^{-2}, S-x u^{-2}\right]} \inf _{s \in\left[t, t+T_{u}\right]} \bar{X}(s) \frac{f_{u}\left(S-x u^{-2}\right)}{f_{u}(s)}>f_{u}\left(S-x u^{-2}\right)\right\} \\
& \leq \mathbb{P}\left\{\sup _{t \in\left[S-x u^{-2}-\lambda u^{-2}, S-x u^{-2}\right]} \inf _{s \in\left[t, t+\left(1-\varepsilon_{1}\right) T u^{-2}\right]} \bar{X}(s) \frac{f_{u}\left(S-x u^{-2}\right)}{f_{u}(s)}>f_{u}\left(S-x u^{-2}\right)\right\} \\
& =\mathbb{P}\left\{\sup _{t \in[0, \lambda]} \inf _{s \in\left[0,\left(1-\varepsilon_{1}\right) T\right]} \bar{X}\left(S+u^{-2} s-u^{-2} t-u^{-2} x\right) \frac{f_{u}\left(S-x u^{-2}\right)}{f_{u}\left(S+u^{-2} s-u^{-2} t-u^{-2} x\right)}>f_{u}\left(S-x u^{-2}\right)\right\} \\
& =\mathbb{P}\left\{\sup _{t \in[0, \lambda]} \inf _{s \in\left[0,\left(1-\varepsilon_{1}\right) T\right]} Y_{u}^{*}(t, s)>f_{u}\left(S-x u^{-2}\right)\right\} \\
& =: \Pi_{0}^{*+}(u) \text {, }
\end{aligned}
$$

and

$$
\Pi_{0}^{*}(u) \geq \mathbb{P}\left\{\sup _{t \in[0, \lambda]} \inf _{s \in\left[0,\left(1+\varepsilon_{1}\right) T\right]} Y_{u}^{*}(t, s)>f_{u}\left(S-x u^{-2}\right)\right\}=: \Pi_{0}^{*-}(u),
$$

where $Y_{u}^{*}(t, s):=\bar{X}\left(S+u^{-2} s-u^{-2} t-u^{-2} x\right) \frac{f_{u}\left(S-x u^{-2}\right)}{f_{u}\left(S+u^{-2} s-u^{-2} t-u^{-2} x\right)},(t, s) \in[0, \lambda] \times\left[0,\left(1+\varepsilon_{1}\right) T\right]$ and $\sigma_{Y_{u}^{*}}^{2}(t, s):=$ $\operatorname{Var}\left(Y_{u}^{*}(t, s)\right)=\left(\frac{f_{u}\left(S-x u^{-2}\right)}{f_{u}\left(S+u^{-2} s-u^{-2} t-u^{-2} x\right)}\right)^{2}$.

Using the similar argumentation as (3.10) in the proof of Theorem 2.1, we have

$$
\lim _{u \rightarrow \infty} \sup _{(t, s) \in[0, \lambda] \times\left[0,\left(1+\varepsilon_{1}\right) T\right]}\left|u^{2}\left(1-\sigma_{Y_{u}^{*}}(t, s)\right)-d(t, s)\right|=0,
$$

with $d(t, s)=\frac{\delta e^{-2 \delta S}}{1-e^{-2 \delta S}}(t-s)$. Moreover, (3.11), (3.12) still hold for $Y_{u}^{*}(t, s)$ and $\left(t_{1}, s_{1}\right),\left(t_{2}, s_{2}\right) \in[0, \lambda] \times\left[0,\left(1+\varepsilon_{1}\right) T\right]$. By Lemma 5.1 in [5] and $\lim _{u \rightarrow \infty} f_{u}(S) / u=1 / \sigma_{X}(S)$, we obtain

$$
\Pi_{0}^{*-}(u) \sim \widetilde{\mathcal{P}}\left(a \lambda, a\left(1+\varepsilon_{1}\right) T\right) \Psi\left(f_{u}\left(S-x u^{-2}\right)\right) \sim e^{-a x} \widetilde{\mathcal{P}}\left(a \lambda, a\left(1+\varepsilon_{1}\right) T\right) \Psi\left(f_{u}(S)\right), u \rightarrow \infty .
$$

Similarly,

$$
\Pi_{0}^{*+}(u) \sim e^{-a x} \widetilde{\mathcal{P}}\left(a \lambda, a\left(1-\varepsilon_{1}\right) T\right) \Psi\left(f_{u}(S)\right), u \rightarrow \infty .
$$

Letting $\varepsilon_{1} \rightarrow 0$ and $\lambda \rightarrow \infty$, we have

$$
\Pi_{0}^{*}(u) \sim e^{-a x} \widetilde{\mathcal{P}}(a T) \Psi\left(f_{u}(S)\right), u \rightarrow \infty .
$$

The above combined with (3.15), (3.16) and (3.17) derives that

$$
\mathbb{P}\left\{\sup _{t \in\left[0, S-x u^{-2}\right]} \inf _{s \in\left[t, t+T_{u}\right]} X_{u}(s)>f_{u}(S)\right\} \sim e^{-a x} \widetilde{\mathcal{P}}(a T) \Psi\left(f_{u}(S)\right), u \rightarrow \infty .
$$

Thus, the claim follows by using the results of Theorem 2.1 and (3.14).

Case $2 \delta=0$ :

$$
\mathbb{P}\left\{u^{2}\left(S+T_{u}-\eta(u)\right)>x \mid \eta(u) \leq S+T_{u}\right\}=\frac{\mathbb{P}\left\{\sup _{t \in\left[0, S-x u^{-2}\right]} \inf _{s \in\left[t, t+T_{u}\right]}(\sigma B(s)-c s)>u\right\}}{\mathbb{P}\left\{\sup _{t \in[0, S]} \inf _{s \in\left[t, t+T_{u}\right]}(\sigma B(s)-c s)>u\right\}}
$$


For $u$ large enough

$$
\mathbb{P}\left\{\sup _{t \in\left[0, S-x u^{-2}\right]} \inf _{s \in\left[t, t+T_{u}\right]}(\sigma B(s)-c s)>u\right\}=\mathbb{P}\left\{\sup _{t \in\left[0, S-x u^{-2}\right]} \inf _{s \in\left[t, t+T_{u}\right]} \tilde{X}_{u}(s)>f_{u}(S)\right\},
$$

with

$$
X(s)=\sigma B(s), \bar{X}(s)=\frac{B(s)}{\sqrt{s}}, f_{u}(s)=\frac{u+c s}{\sigma \sqrt{s}} \text { and } \tilde{X}_{u}(s)=\bar{X}(s) \frac{f_{u}(S)}{f_{u}(s)} .
$$

Set $\rho(u)=\left(\frac{\ln u}{u}\right)^{2}$. For any $\lambda>0$, Bonferroni inequality yields

$$
\widetilde{\Pi}_{0}(u) \leq \mathbb{P}\left\{\sup _{t \in\left[0, S-x u^{-2}\right]} \inf _{s \in\left[t, t+T_{u}\right]} \widetilde{X}_{u}(s)>f_{u}(S)\right\} \leq \widetilde{\Pi}_{0}(u)+\widetilde{\Pi}_{1}(u)+\widetilde{\Pi}_{2}(u),
$$

where

$$
\begin{aligned}
& \widetilde{\Pi}_{0}(u)=\mathbb{P}\left\{\sup _{t \in\left[S-x u^{-2}-\lambda u^{-2}, S-x u^{-2}\right]} \inf _{s \in\left[t, t+T_{u}\right]} \widetilde{X}_{u}(s)>f_{u}(S)\right\}, \\
& \widetilde{\Pi}_{1}(u)=\mathbb{P}\left\{\sup _{t \in[0, S-\rho(u)]} \inf _{s \in\left[t, t+T_{u}\right]} \widetilde{X}_{u}(s)>f_{u}(S)\right\}, \\
& \widetilde{\Pi}_{2}(u)=\mathbb{P}\left\{\sup _{t \in\left[S-\rho(u), S-x u^{-2}-\lambda u^{-2}\right]} \inf _{s \in\left[t, t+T_{u}\right]} \widetilde{X}_{u}(s)>f_{u}(S)\right\} .
\end{aligned}
$$

Notice that for $u$ large enough

$$
\begin{aligned}
\mathbb{E}\left\{\left(\widetilde{X}_{u}\left(t_{1}\right)-\widetilde{X}_{u}\left(t_{2}\right)\right)^{2}\right\} & =\frac{1}{S} \mathbb{E}\left\{\left(\frac{u+c S}{u+c t_{1}} B\left(t_{1}\right)-\frac{u+c S}{u+c t_{2}} B\left(t_{2}\right)\right)^{2}\right\} \\
& \leq \mathbb{C}_{12} \mathbb{E}\left\{\left(B\left(t_{1}\right)-B\left(t_{2}\right)\right)^{2}\right\}+\mathbb{C}_{13}\left(\frac{u+c S}{u+c t_{1}}-\frac{u+c S}{u+c t_{2}}\right)^{2} \\
& \leq \mathbb{C}_{14}\left|t_{1}-t_{2}\right|, \quad t_{1}<t_{2}, t_{1}, t_{2} \in(0, S]
\end{aligned}
$$

and

$$
\sup _{t \in[0, S-\rho(u)]} \operatorname{Var}\left(\widetilde{X}_{u}(t)\right)=\sup _{t \in[0, S-\rho(u)]}\left(\frac{f_{u}(S)}{f_{u}(t)}\right)^{2}=\frac{f_{u}^{2}(S)}{f_{u}^{2}(S-\rho(u))},
$$

where we use the fact that $f_{u}(t)$ is a decreasing function for $t \in[0, S]$ when $u$ large enough.

Moreover,

$$
\begin{gathered}
1-\frac{f_{u}(S)}{f_{u}(S-t)} \sim \frac{1}{2 S} t, \quad t \rightarrow 0, \\
\inf _{1 \leq k \leq N(u)} f_{u}\left(S-k \lambda u^{-2}\right) \rightarrow \infty, u \rightarrow \infty,
\end{gathered}
$$

and for $t_{1}<t_{2}, t_{1}, t_{2} \in[0, S]$,

$$
r_{\widetilde{X}}\left(t_{1}, t_{2}\right):=\mathbb{E}\left\{\bar{X}\left(t_{1}\right) \bar{X}\left(t_{2}\right)\right\}=\sqrt{\frac{t_{1}}{t_{2}}} .
$$

Then

$$
\begin{aligned}
& \lim _{u \rightarrow \infty} \sup _{1 \leq k \leq N(u)} \sup _{\substack{t_{1} \neq t_{2}, t_{1}, t_{2} \in[0, \lambda]}}\left|f_{u}^{2}\left(S-k \lambda u^{-2}\right) \frac{\operatorname{Var}\left(\bar{X}\left(S-u^{-2} t_{1}\right)-\bar{X}\left(S-u^{-2} t_{2}\right)\right)}{2 b\left|t_{1}-t_{2}\right|}-1\right| \\
& \quad=\lim _{u \rightarrow \infty} \sup _{1 \leq k \leq N(u)} \sup _{\substack{t_{1} \neq t_{2}, t_{1}, t_{2} \in[0, \lambda]}}\left|f_{u}^{2}\left(S-k \lambda u^{-2}\right) \frac{2-2 r_{\tilde{X}}\left(S-u^{-2} t_{1}, S-u^{-2} t_{2}\right)}{2 b\left|t_{1}-t_{2}\right|}-1\right|=0,
\end{aligned}
$$


where $b=\frac{1}{2 \sigma^{2} S^{2}}$, and

$$
\begin{aligned}
& \sup _{1 \leq k \leq N(u)} \sup _{\substack{\left|t_{1}-t_{2}\right|<\varepsilon \\
t_{1}, t_{2} \in[0, \lambda]}} f_{u}^{2}\left(S-k \lambda u^{-2}\right) \mathbb{E}\left\{\left(\bar{X}\left(S-u^{-2} t_{1}\right)-\bar{X}\left(S-u^{-2} t_{2}\right)\right) \bar{X}(S)\right\} \\
& \leq \mathbb{C}_{15} u^{2} \sup _{\substack{\left|t_{1}-t_{2}\right|<\varepsilon \\
t_{1}, t_{2} \in[0, \lambda]}}\left|r_{\widetilde{X}}\left(S-u^{-2} t_{1}, S\right)-r_{\widetilde{X}}\left(S-u^{-2} t_{2}, S\right)\right| \\
& \leq \mathbb{C}_{16} u^{2} \sup _{\left|t_{1}-t_{2}\right|<\varepsilon}\left|\sqrt{S-u^{-2} t_{1}}-\sqrt{S-u^{-2} t_{2}}\right| \\
& \quad \mathbb{C}_{17} \sup _{\substack{t_{1}, t_{2} \in[0, \lambda] \\
\left|t_{1}-t_{2}\right|<\varepsilon \\
t_{1}, t_{2} \in[0, \lambda]}}\left|t_{1}-t_{2}\right| \rightarrow 0, u \rightarrow \infty, \varepsilon \rightarrow 0 .
\end{aligned}
$$

By Theorem 8.1 in [17] and Lemma 5.3 in [2], using the similar argumentation as in the proof of Theorem 2.1, we derive

$$
\widetilde{\Pi}_{1}(u)+\widetilde{\Pi}_{2}(u)=o\left(\Psi\left(f_{u}(S)\right)\right), u \rightarrow \infty, \lambda \rightarrow \infty .
$$

Next we give the asymptotic behavior of $\widetilde{\Pi}_{0}(u)$ as $u \rightarrow \infty$. For any $\varepsilon_{1}>0$ and $u$ large enough

$$
\begin{aligned}
\widetilde{\Pi}_{0}(u) & =\mathbb{P}\left\{\sup _{t \in\left[S-x u^{-2}-\lambda u^{-2}, S-x u^{-2}\right]} \inf _{s \in\left[t, t+T_{u}\right]} \bar{X}(s) \frac{f_{u}(S)}{f_{u}(s)}>f_{u}(S)\right\} \\
& =\mathbb{P}\left\{\inf _{t \in\left[S-x u^{-2}-\lambda u^{-2}, S-x u^{-2}\right]} \bar{X} \in\left[(s) \frac{f_{u}\left(S-x u^{-2}\right)}{f_{u}(s)}>T_{u}\left(S-x u^{-2}\right)\right\}\right. \\
& \leq \mathbb{P}\left\{\sup _{t \in[0, \lambda]} \inf _{s \in\left[0,\left(1-\varepsilon_{1}\right) T\right]} \widetilde{Y}_{u}(t, s)>f_{u}\left(S-x u^{-2}\right)\right\} \\
& =: \widetilde{\Pi}_{0}^{+}(u)
\end{aligned}
$$

and

$$
\widetilde{\Pi}_{0}(u) \geq \mathbb{P}\left\{\sup _{t \in[0, \lambda]} \inf _{s \in\left[0,\left(1+\varepsilon_{1}\right) T\right]} \widetilde{Y}_{u}(t, s)>f_{u}\left(S-x u^{-2}\right)\right\}=: \widetilde{\Pi}_{0}^{-}(u),
$$

where $\widetilde{Y}_{u}(t, s):=\bar{X}\left(S+u^{-2} s-u^{-2} t-u^{-2} x\right) \frac{f_{u}\left(S-x u^{-2}\right)}{f_{u}\left(S+u^{-2} s-u^{-2} t-u^{-2} x\right)}$, for $(t, s) \in[0, \lambda] \times\left[0,\left(1+\varepsilon_{1}\right) T\right]$.

Using the similar argumentation as (3.10), (3.11) and (3.12) in the proof of Theorem 2.1, we obtain that

$$
\lim _{u \rightarrow \infty} \sup _{(t, s) \in[0, \lambda] \times\left[0,\left(1+\varepsilon_{1}\right) T\right]}\left|u^{2}\left(1-\sigma_{\widetilde{Y}_{u}}(t, s)\right)-\widetilde{d}(t, s)\right|=0,
$$

with $\widetilde{d}(t, s)=\frac{1}{2 S}(t-s)$ and $\sigma_{\widetilde{Y}_{u}}(t, s):=\sqrt{\operatorname{Var}\left(\widetilde{Y}_{u}(t, s)\right)}$,

$$
\lim _{u \rightarrow \infty} u^{2} \operatorname{Var}\left(\widetilde{Y}_{u}\left(t_{1}, s_{1}\right)-\widetilde{Y}_{u}\left(t_{2}, s_{2}\right)\right)=\frac{1}{S} \operatorname{Var}\left(B\left(s_{1}-t_{1}\right)-B\left(s_{2}-t_{2}\right)\right),
$$

and for some constant $G$ and all $u$ large enough

$$
u^{2} \operatorname{Var}\left(\widetilde{Y}_{u}\left(t_{1}, s_{1}\right)-\widetilde{Y}_{u}\left(t_{2}, s_{2}\right)\right) \leq G\left(\left|t_{1}-t_{2}\right|+\left|s_{1}-s_{2}\right|\right)
$$

uniformly for $\left(t_{1}, s_{1}\right),\left(t_{2}, s_{2}\right) \in[0, \lambda] \times\left[0,\left(1+\varepsilon_{1}\right) T\right]$.

By Lemma 5.1 in [5] and $\lim _{u \rightarrow \infty} f_{u}(S) / u=\frac{1}{\sigma \sqrt{S}}$, we obtain

$$
\widetilde{\Pi}_{0}^{-}(u) \sim \widetilde{\mathcal{P}}\left(b \lambda, b\left(1+\varepsilon_{1}\right) T\right) \Psi\left(f_{u}\left(S-x u^{-2}\right)\right) \sim e^{-b x} \widetilde{\mathcal{P}}\left(b \lambda, b\left(1+\varepsilon_{1}\right) T\right) \Psi\left(f_{u}(S)\right), u \rightarrow \infty .
$$

Similarly,

$$
\widetilde{\Pi}_{0}^{+}(u) \sim e^{-b x} \widetilde{\mathcal{P}}\left(b \lambda, b\left(1-\varepsilon_{1}\right) T\right) \Psi\left(f_{u}(S)\right), u \rightarrow \infty .
$$


Letting $\varepsilon_{1} \rightarrow 0$ and $\lambda \rightarrow \infty$, we have

$$
\widetilde{\Pi}_{0}(u) \sim e^{-b x} \widetilde{\mathcal{P}}(b T) \Psi\left(f_{u}(S)\right), u \rightarrow \infty .
$$

The above combined with (3.19) and (3.22) leads to

$$
\mathbb{P}\left\{\sup _{t \in\left[0, S-x u^{-2}\right]} \inf _{s \in\left[t, t+T_{u}\right]} \widetilde{X}_{u}(s)>f_{u}\left(S_{x}(u)\right)\right\} \sim e^{-b x} \widetilde{\mathcal{P}}(b T) \Psi\left(f_{u}(S)\right), u \rightarrow \infty
$$

Using the above asymptotic equality and b) of Remarks 2.2, we obtain the results.

Acknowledgement: Thanks to Swiss National Science Foundation grant no. 200021-166274.

\section{REFERENCES}

[1] L. Bai, K. Dȩbicki, E. Hashorva, and L. Luo. On generalised Piterbarg constants. Manuscript, 2016.

[2] K. Dębicki, E. Hashorva, and P. Liu. Ruin probabilities and passage times of $\gamma$-reflected Gaussian processes with stationary increments. http://arXiv.org/abs/1511.09234, 2015.

[3] K. Dẹbicki, E. Hashorva, and L. Ji. Gaussian risk model with financial constraints. Scandinavian Actuarial Journal, 2015(6):469-481, 2015.

[4] K. Dẹbicki, E. Hashorva, and L. Ji. Parisian ruin of self-similar Gaussian risk processes. Journal of Applied Probability, 52(3):688-702, 2015.

[5] K. Dẹbicki, E. Hashorva, and L. Ji. On Parisian ruin over a finite-time horizon. Science China Mathematics, $59(3): 557-572,2016$.

[6] K. Dẹbicki and M. Mandjes. Exact overflow asymptotics for queues with many Gaussian inputs. Journal of Applied Probability, 40(3):704-720, 2003.

[7] G. Deelstra. Remarks on boundary crossing result for Brownian motion. Blätter der DGVFM, 21(4):449-456, 1994.

[8] A. B. Dieker. Extremes of Gaussian processes over an infinite horizon. Stochastic Processes and their Applications, 115(2):207-248, 2005.

[9] D. C. Emanuel, J. M. Harrison, and A. J. Taylor. A diffusion approximation for the ruin function of a risk process with compounding assets. Scandinavian Actuarial Journal, 1975(4):240-247, 1975.

[10] P. Embrechts, C. Klüppelberg, and T. Mikosch. Modelling extremal events. Applications of Mathematics (New York). Springer-Verlag, Berlin, 1997.

[11] J. M. Harrison. Ruin problems with compounding assets. Stochastic Processes and their Applications, 5(1):67-79, 1977.

[12] E. Hashorva and L. Ji. Approximation of passage times of $\gamma$-reflected processes with fBm input. Journal of Applied Probability, 51(3):713-726, 2015.

[13] E. Hashorva and L. Ji. Piterbarg theorems for chi-processes with trend. Extremes, 18(1):37-64, 2015.

[14] X. He and Y. Hu. Ruin probability for the integrated Gaussian process with force of interest. Journal of Applied Probability, 44:685-694, 2007.

[15] J. Hüsler and V. I. Piterbarg. Extremes of a certain class of Gaussian processes. Stochastic Processes and their Applications, 83(2):257-271, 1999.

[16] J. Hüsler and V. I. Piterbarg. A limit theorem for the time of ruin in a Gaussian ruin problem. Stochastic Processes and their Applications, 118(11):2014-2021, 2008.

[17] V. I. Piterbarg. Asymptotic methods in the theory of Gaussian processes and fields, volume 148 of Translations of Mathematical Monographs. American Mathematical Society, Providence, RI, 1996. 
[18] T. Rolski, H. Schmidli, V. Schmidt, and J. Teugels. Stochastic processes for insurance and finance. John Wiley \& Sons, 2009.

Long Bai, Department of Actuarial Science, University of Lausanne, UNiL-Dorigny, 1015 Lausanne, Switzerland

E-mail address: Long.Bai@unil.ch

Li Luo, Department of Actuarial Science, University of Lausanne, UniL-Dorigny, 1015 Lausanne, Switzerland and School of Mathematical Sciences, Nankai University, Tianjin 300071, P.R. China

E-mail address: Li.Luo@unil.ch 